\title{
SIMES: A Simulator for Hybrid Electrical Energy Storage Systems
}

\author{
Siyu Yue, Di Zhu, Yanzhi Wang, and Massoud Pedram \\ University of Southern California, CA, USA \\ \{siyuyue, dizhu, yanzhiwa, pedram\}@usc.edu
}

\author{
Younghyun Kim, and Naehyuck Chang \\ Seoul National University, Korea \\ \{yhkim,naehyuck\}@elpl.snu.ac.kr
}

\begin{abstract}
State-of-the-art electrical energy storage (EES) systems are mainly homogeneous, i.e., they consist of a single type of EES elements. None of the existing EES elements is capable of simultaneously fulfilling all the desired features of an ideal EES system, e.g., high charge/discharge efficiency, high energy density, low cost per unit capacity, long cycle life. A novel technology, i.e., a hybrid EES system that employs heterogeneous EES elements organized in a hierarchy of storage banks and linked by appropriate charge transfer interconnects, has shown great promise in overcoming the aforesaid limitations of conventional EES systems. However, the widespread adoption/deployment of hybrid EES systems is hampered by lack of a hybrid EES system simulator. This paper thus presents SIMES, a powerful and scalable simulator for hybrid EES systems, which provides fast and accurate system simulations, while accounting for key characteristics of various EES elements, power converters, charge transfer interconnect schemes, etc. Experimental results on two different applications (one targeting load shifting for households, the other related to battery rate capacity effect minimization in portable electronic devices) demonstrate the value and usefulness of SIMES for designing energy-aware facilities and products.
\end{abstract}

\section{Keywords}

Hybrid Electrical Energy Storage System, Simulator

\section{Introduction}

An electrical energy storage (EES) system is an energy reservoir that stores energy electrically and supplies energy when necessary. It is considered to store a higher grade of energy compared to other energy storage systems since electrical energy has a greater capability in controlling and converting to other forms of energy [1]. Applications of EES systems range from storing and supplying power for the grid to providing power for portable electronics.

Performance metrics describing an EES system include capital cost, cycle efficiency, energy density, power capacity, cycle life, and environmental effects. State-of-the-art EES system deployments are mainly homogeneous, i.e., they consist of a single type of EES element, such as Lithium-ion (Li-ion) batteries, lead-acid batteries or supercapacitors. Unfortunately, none of the existing EES elements can simultaneously achieve high performance in all of the above performance metrics. For example, lead-acid batteries have low cost per unit capacity, but their energy density is much smaller than that of Li-ion batteries. Li-ion batteries and lead-acid batteries both suffer from efficiency degradation due to the rate capacity effect, which is negligible in supercapacitors [20]. In contrast, supercapacitors exhibit severe self-discharge and have high cost [2].

A novel technology aiming at overcoming the above limitations, the hybrid EES (HEES) system, is proposed in recent years and gaining popularity [2]. Analogy is made to the computer memory hierarchy, where the memory system takes advantage of the strengths of each individual element type to achieve short access time, high density and low cost simultaneously. A HEES system is also comprised of several heterogeneous EES elements and seeks to exploit the strengths of each type of EES elements while hiding their weaknesses. Recent works on design considerations and con- trol policies to realize the potential benefits of HEES systems have been proposed in [3], [5]-[11] and [19].

There are three key problems in the design and management of HEES systems, namely charge allocation, charge migration, and charge replacement. Charge allocation is the problem of distributing the incoming power to different destination EES banks [7]. References [8] and [9] discuss the charge migration problem of transferring electrical charge from source banks to destination banks so as to improve the overall efficiency. Charge replacement [10] is the management of discharge currents of EES banks to serve a given load demand. These three problems can be combined into a joint optimization problem, where the HEES system is connected to both external sources and loads, and an optimal management policy is required for simultaneous charge allocation, migration and replacement [2][13].

A lot of recent research work focuses on different applications of the HEES system. Shin et al. propose in [12] to use a batterysupercapacitor hybrid system for high-rate pulsed load applications where the supercapacitor acts as a low pass filter that prunes out rapid load changes, which alleviates the rate capacity effect of batteries and improves the system's energy efficiency. Xie et al. extend this idea to both charge allocation and charge replacement to minimize the State-of-Health degradation rate of batteries [14] Mirhoseini et al. consider a similar setting where the batterysupercapacitor hybrid system is used to power a load device to accomplish multiple schedulable tasks [4]. An online learningbased control policy for a similar problem is proposed in [16] Sharma et al. propose the application of a hybrid system consisting of supercapacitors, flywheels and batteries to accommodate base load, peak load and instantaneous load in a microgrid [17]. Cao et al. propose a battery-supercapacitor hybrid system for electric vehicles in [18].

The vast number of research topics and issues on the design and management of HEES systems for different applications motivate the deployment of a powerful and scalable simulation platform. Therefore, we present SIMES, a simulator for HEES systems. The main contributions of SIMES include:

1) SIMES is the first simulation platform for HEES systems, which can perform fast and accurate simulation for different scales of HEES systems in a wide range of applications.

2) SIMES implements a set of models for common components in a HEES system, most of which are calibrated based on the measurement data of actual hardware.

3) SIMES handles different power source and load profiles and let users easily explore different configuration of HEES systems, such as EES element types, bank sizes, etc.

4) SIMES has great extensibility. It provides a convenient interface for users to implement their own component models as $\mathrm{C}++$ classes and add them to SIMES.

The rest of this paper is organized as follows. Section 2 introduces the background of HEES systems. Section 3 and Section 4 present the overview and implementation details of SIMES, respectively. In Section 5, we first validate SIMES's accuracy by comparing simulation results with a HEES prototype system's measurement. Then we demonstrate two example usage of SIMES in both household and mobile applications. Section 6 concludes this paper. 


\section{HEES System Background}

There are different architectures for a HEES system. A single Charge Transfer Interconnect (CTI)-based HEES system is shown in Figure 1 to deliver the concept of HEES systems. SIMES also accommodates various CTI architectures [19]. Below is a brief introduction of the basic components in the HEES systems.

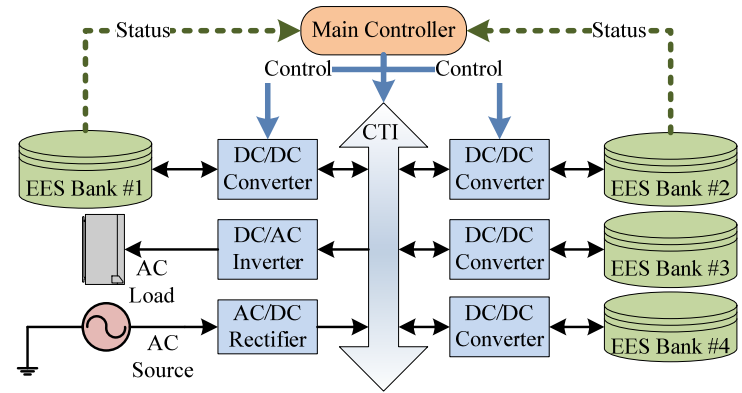

Figure 1. An example of HEES system structure.

1) Power Sources

A HEES system deposits energy from power sources, either AC or DC. Common power sources for a HEES system include the AC grid, PV cells, or wind turbines.

2) Storage Banks

Storage banks refer to the EES elements in the system. An EES bank contains $N$ in series by $M$ in parallel homogenous cells. Different banks may be heterogeneous, which have different characteristics such as cycle efficiency, energy density, power density, cycle life, etc. Electrical energy stored in the system is distributed among different banks according to some quality-of-service requirements and optimization goals of the joint management policy.

3) Load Devices

Load devices are powered by the HEES system and can be AC or DC. Different types of loads include household electrical appliances, electric vehicles, portable devices, etc.

4) Charge transfer interconnect (CTI)

A CTI is a medium over which electric current flow forms among banks, load devices and power sources. The architecture (topology) of the CTI has a significant impact on the performance of the HEES system, especially for charge transfer efficiency. A single shared-bus CTI architecture is low cost and suitable to accommodate a small number of banks [7]. However, multiple concurrent charge transfers should be performed through the shared path with the same voltage, which may significantly degrade the efficiency with a large number of banks [19]. On the other hand, multiple CTI buses and a networked CTI are more scalable than the single-bus CTI because they may provide more independent paths for concurrent charge transfers [19].

\section{5) Power Converters}

Power converters include DC-DC converters between the CTI and DC load devices, DC-AC inverters between the CTI and AC load devices, AC-DC rectifier between the CTI and AC power sources, chargers between the CTI and the EES elements in a bank, etc. Their input or output currents $I_{\text {in }}, I_{\text {out }}$ can be regulated through internal feedback control, and their set points are determined by the main controller.

\section{6) Main Controller}

The operation of the HEES system is monitored and controlled by a global main controller. It collects information from other components and sends commands to the converters to control their output/input current together with voltage levels on the CTIs.
Components 1) through 5) are physical components in the system while 6) is usually a software implemented in the supervisor controller. The connections between all the physical components in the HEES system are modeled as a graph. Sources, banks and loads in this graph are nodes with degree of one. CTIs are nodes with degree of two or more. Power converters are the edges that connect the nodes together.

\section{Simulator Overview}

SIMES proposed in this paper is a simulation platform for HEES systems, which can be applied to different applications. The input of SIMES is an XML file describing the structure of the HEES system and specifying the simulation environment. SIMES monitors the properties of various components in the HEES system, such as the CTI voltage, the load energy consumption and the battery SoCs, and stores them to a text file.

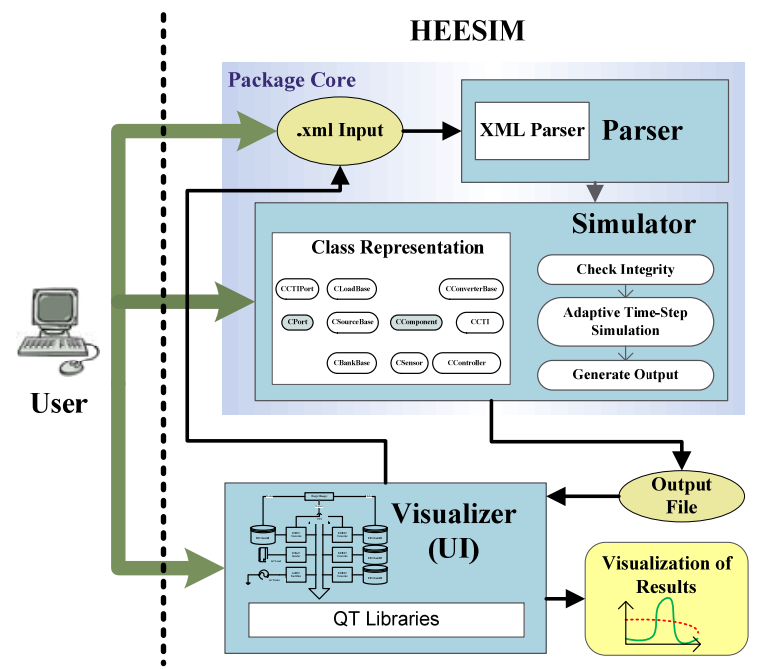

Figure 2. SIMES overview.

SIMES consists of three main modules, namely Parser, Simulator, and Visualizer (UI), as depicted in Figure 2. The entire simulation flow consists of four steps. In the beginning the input XML file is written by the user or automatically generated by Visualizer. Next, Parser reads the input file and calls the interface provided by Simulator to construct the HEES system. Then Simulator simulates the operation of the HEES system. Simulator can work in two modes: in the autonomous mode, it executes the commands provided by Parser whereas in interaction mode it accepts user input from the terminal during simulation. Finally, the output file is generated and shown in Visualizer.

\section{Simulator Implementation}

SIMES is implemented using $\mathrm{C}++$. SIMES itself does not try to provide an extensive collection of all the component models. Instead, SIMES provides a convenient interface so that users can implement their own component models as $\mathrm{C}++$ classes derived from the base classes and add them by including their constructor functions in the corresponding factory methods.

\subsection{Component Modeling}

We implement each type of component in the HEES system as a $\mathrm{C}++$ class. Among these classes, CPort and CComponent are the two virtual base classes that are used to derive other classes.

CPort is the abstraction of the connection between any component and a converter. It provides the interface which defines the I-V characteristic curve of that connection: 


$$
V=f_{p}(I, t)
$$

where $V$ is the terminal voltage, $I$ is the port current and $t$ is the present time of simulation.

CComponent serves as the base class of all components in the HEES system. It mainly provides timing and property interfaces. The timing interface defines the functions which can be called by the simulator to simulate the operation of a component during a certain time span. The property interface is used to set/get a certain property or parameter of a component.

The storage banks modeled in SIMES include Li-ion batteries, lead-acid batteries, and supercapacitors. We mainly consider the following characteristics of storage banks in SIMES.

1) Open Circuit Voltage (OCV) as a function of State-of-Charge (SoC): $V_{O C}(S o C)$. SoC of a battery is defined as the ratio of the remaining charge to its full charge capacity (FCC) [20].

2) Internal Resistance as a function of SoC during charging and discharging process: $R_{\text {int }, c}(\mathrm{SoC}), R_{\text {int }, d}(\mathrm{SoC})$. Figure 3 shows the measured $V_{O C}(S o C), R_{\text {int }, c}(S o C)$ and $R_{\text {int }, d}(\operatorname{SoC})$ curves of a lead-acid battery [24].
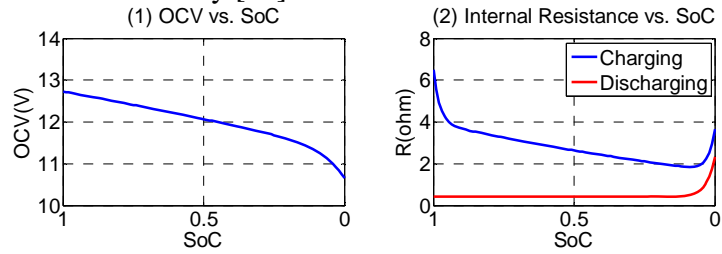

Figure 3. OCV and IR vs. SoC curve.

3) Rate Capacity Effect: Rate capacity effect captures the fact that the actual charge loss rate $I_{\text {loss }}$ inside a battery is a superlinear function of its discharge current $I_{\text {disch }}$ [20]. The discharge efficiency $\eta$, defined as $I_{\text {disch }} / I_{\text {loss }}$, is a monotonically decreasing function of $I_{\text {disch }}$, denoted by $\eta\left(I_{\text {disch }}\right)$. Peukert's law is an empirical equation that evaluates the rate capacity effect:

$$
\eta\left(I_{\text {disch }}\right)=\frac{k}{\left(I_{\text {disch }}\right)^{\alpha}}
$$

where $k, \alpha$ are constants. Figure 4 shows the measured result of discharge efficiency of the same lead-acid battery and the fitted curved using Peukert's law.

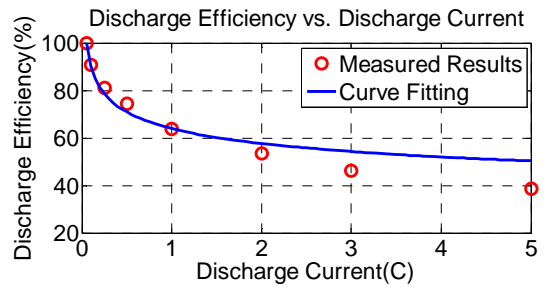

Figure 4. Discharge efficiency vs. discharge current ${ }^{1}$.

4) Self-Discharge Effect: Self discharge effect is not prominent in ordinary batteries, but is an important issue in supercapacitors. A supercapacitor generally loses $20 \% \sim 40 \%$ of its stored energy during one day even if it is not connected to any load device [2]. The supercapacitor voltage decay after $\Delta t$ time is:

$$
V_{S}(t+\Delta t)=V_{S}(t) e^{-\Delta t / \tau}
$$

where $\tau$ denotes the self-discharge time constant.

5) State-of-Health (SoH) Degradation: $\mathrm{SoH}$ is defined as the ratio of FCC of a cycle-aged EES element to its initial capacity (i.e. the FCC of the same fresh new element). We adopt the SoH model of Li-ion batteries proposed in [14].

${ }^{1}$ For a given battery, a current of $1 \mathrm{C}$ equals to the capacity of that battery (in Ah) divided by one hour.
In SIMES power converters are modeled by their input-output relationship. Let $V_{\text {out }}, I_{\text {out }}, V_{\text {in }}$ and $I_{\text {in }}$ denote the output voltage and current, input voltage and current of a power converter, respectively. The following interface should be provided (these two functions should be consistent with each other):

$$
\begin{aligned}
& I_{\text {out }}=f_{c 1}\left(V_{\text {out }}, V_{\text {in }}, I_{\text {in }}, t\right) \\
& I_{\text {in }}=f_{c 2}\left(V_{\text {out }}, V_{\text {in }}, I_{\text {out }}, t\right)
\end{aligned}
$$

The above interface determines the power conversion efficiency of a power converter, which is defined as follows:

$$
\eta_{\text {conv }}=\frac{P_{\text {out }}}{P_{\text {in }}}=\frac{V_{\text {out }} I_{\text {out }}}{V_{\text {in }} I_{\text {in }}}
$$

For example, Figure 5 shows the measured data of the efficiency of LTC3789 [15] as a function of input and output voltages with two different output currents. In SIMES such information can be provided to a power converter class as a three dimensional lookup table.
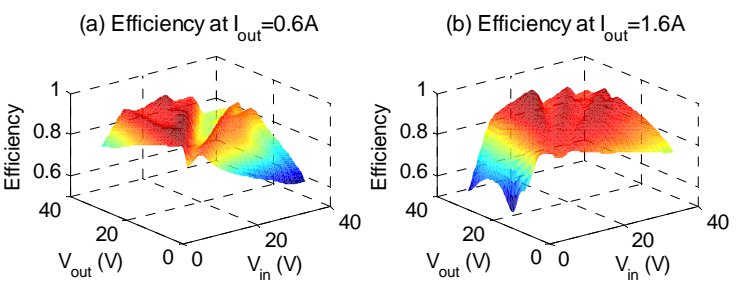

Figure 5. Converter efficiency at different output currents.

The HEES prototype maintains CTI stability with a capacitor of around $100 \mathrm{mF}$ electrolytic capacitance. We thus model the CTI with a capacitor. For each CTI, one power converter is designated as the voltage regulator to maintain the voltage level of that CTI.

\subsection{Module Breakdown}

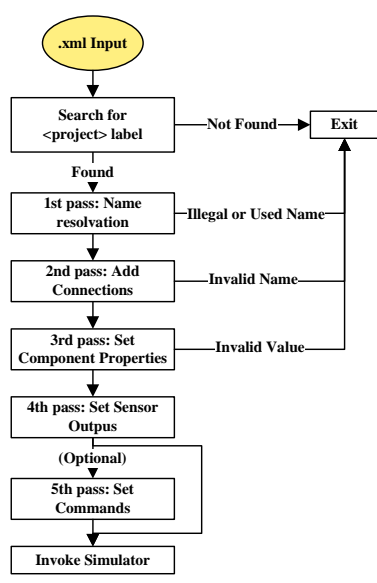

(a) Parser workflow

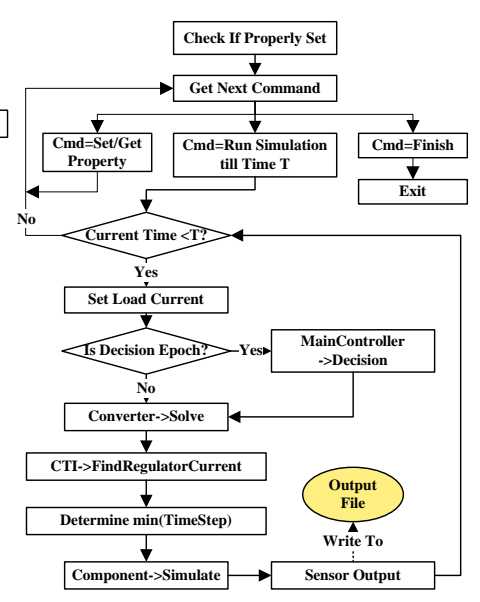

(b) Simulator workflow
Figure 6. Main workflow of Parser and Simulator.

\subsubsection{Parser}

The HEES system description file is written in XML. Parser uses TinyXML [25], an open source lightweight C++ XML parser, to parse the input XML file. Parser uses the interface provided by Simulator to construct the HEES system by instantiating $\mathrm{C}++$ classes corresponding to each component and setting their properties. Parser also extracts the commands to be executed by Simulator if it is set to work in autonomous mode. The main flow of the parsing process is shown in Figure 6 (a).

The input file is scanned a few times before parsing is complete. In the first pass, Parser resolves names of all components and in- 
stantiates the corresponding $\mathrm{C}++$ classes according to their derived type. The connections between converters and other components are set up in the second pass. In the third pass, Parser sets the properties of each component. In the fourth pass, values that should be outputted are specified. Commands are parsed in the fifth pass.

\subsubsection{Simulator}

After Parser parses the input file and instantiates the $\mathrm{C}++$ classes, Simulator will run adaptive time-step simulation. The main workflow of Simulator is shown in Figure 6 (b). Simulator currently supports the following types of commands: i) Run the simulation till a user-defined time $T$; ii) Set/get the values of each component's property; iii) Finish simulation.

Simulator starts processing commands after checking whether the HEES system is properly set. If the command is to run simulation till time T, Simulator will enter the main loop to perform simulation. In each loop iteration, Simulator will by default satisfy load demand through setting the load current unless the main controller overwrites this decision later. At each decision epoch, the main controller collects information about the status of all other components, makes decisions and sends commands to the converters to set their input or output current. Setting the current at only one side (input/output) of each converter is sufficient since Simulator will invoke the converter's Solve function to determine the current and voltage of both sides.

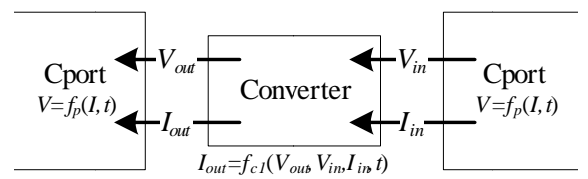

Figure 7. Converter's solve function.

The converter's Solve function works as follows: Suppose $I_{i n}$ is set by the main controller as in Figure 7. $V_{\text {in }}$ can be directly obtained by equation (1). Therefore we only need to determine $I_{\text {out }}$ and $V_{\text {out }}$ through equation (1) and (4) in an iterative fashion. This iteration method is shown in the following pseudo code: (EPS is predefined according to precision requirements, e.g., $10^{-7}$ )

$$
\begin{aligned}
& \text { Count }=0 \\
& I_{\text {out }}=0 \\
& I_{\text {out_pre }}=100 \\
& \text { while Count }<\operatorname{MaxCount} \text { and abs }\left(I_{\text {out }}-I_{\text {out_pre }}\right)>\text { EPS } \\
& \quad \text { Count }++ \\
& \quad I_{\text {out_pre }}=I_{\text {out }} \\
& \quad V_{\text {out }}=\text { CPort.fp }\left(I_{\text {out }}, t\right) \\
& \quad I_{\text {out }}=\text { CConverter.fc } 1\left(V_{\text {out }}, V_{\text {in }}, I_{\text {in }}, t\right)
\end{aligned}
$$

After calling the Solve function of all the converters, the voltage and current of each port is set. CTI's FindRegulatorCurrent function is then invoked to adjust the output current of its voltage regulator based on the difference between the actual CTI voltage and the target CTI voltage set by the main controller. This process is demonstrated in the following pseudo code:

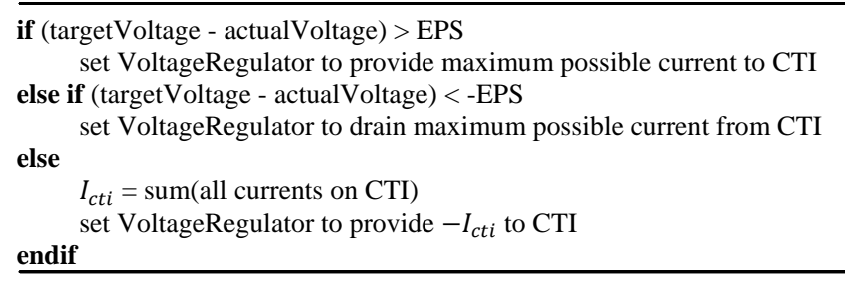

After all the voltages and currents have been set, the minimum time-step is determined by inquiring on each component's acceptable time-step with regard to the predefined precision requirements.
The operation of each component during this time-step is then simulated. Sensor output is recorded in the last step.

\subsubsection{Visualizer}

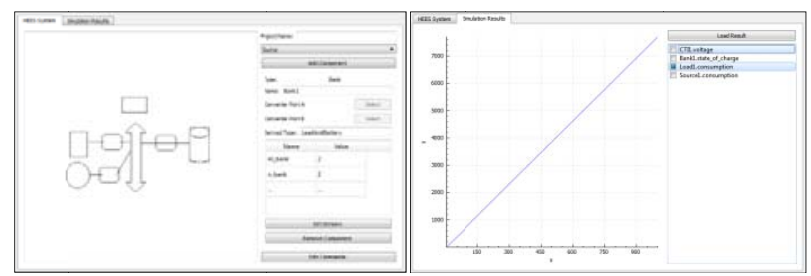

Figure 8. Screenshots of Visualizer.

Visualizer is implemented using Qt 5.0 libraries [26]. It is a standalone application which is not required for HEES system simulation. Visualizer has two functionalities as shown in Figure 8. First, users can use it to directly "draw" the HEES system (by adding the components, setting their properties and showing how they are connected to each other) instead of taking the trouble to write the input XML file. Second, it can plot the simulation results. QCustomPlot [27] is used for the plotting module.

\section{Simulator Evaluation}

\subsection{Validation of SIMES}

Figure 9 shows a HEES system prototype, which is connected to the grid and can be used for household applications. The system consists of a Li-ion battery bank, a lead-acid battery bank and a supercapacitor bank. We conduct measurement on this HEES system prototype and configure SIMES to run the same simulation to validate its accuracy.

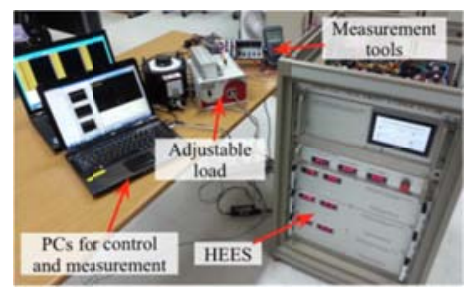

Figure 9. HEES system prototype for measurement.

\subsubsection{Prototype Hardware Measurement}

We measure the prototype and collect data for half an hour. The HEES system is connected to the grid and an adjustable load. We use PCs to control the CTI voltage and input/output current of all the power converters. During the measurement, the Li-ion battery bank only discharges, the lead-acid battery bank and the supercapacitor bank get charged and discharged alternatively (positive current means charging). Standard data acquisition (DAQ) products are used to collect data from the measurement.

\subsubsection{Simulator Setup}

The CTI voltage and terminal currents of all the banks over time serve as the input data of SIMES to conduct simulation. A main controller class is implemented in SIMES to consume these data and set the target CTI voltage together with the input/output current of all the power converters. Simulator simulates the operation of the entire HEES system and outputs the CTI voltage and terminal voltages of all EES banks over time.

\subsubsection{Simulation Results}

The runtime of the simulation is 1.1 s compared to the actual measurement which takes $1 / 2$ hour. Figure 10 shows comparison of the measured data and SIMES outputs. When calculating the average simulation error, we exclude the initial period (0 to around $300 \mathrm{~s})$ of 
the Li-ion battery bank and the lead-acid battery bank because the hardware measurement setup cannot report accurate voltage when the current is zero. The average simulation error of the CTI voltage, closed circuit voltage (CCV) of Li-ion battery bank, lead-acid battery bank and supercapacitor bank is $0.05 \%, 0.1 \%, 2.3 \%$ and $1.7 \%$, respectively. The simulation results did not match well for the leadacid bank because the battery used in the HEES prototype is an aged battery whose state-of-health and charge/discharge characteristics are difficult to quantify and model precisely.
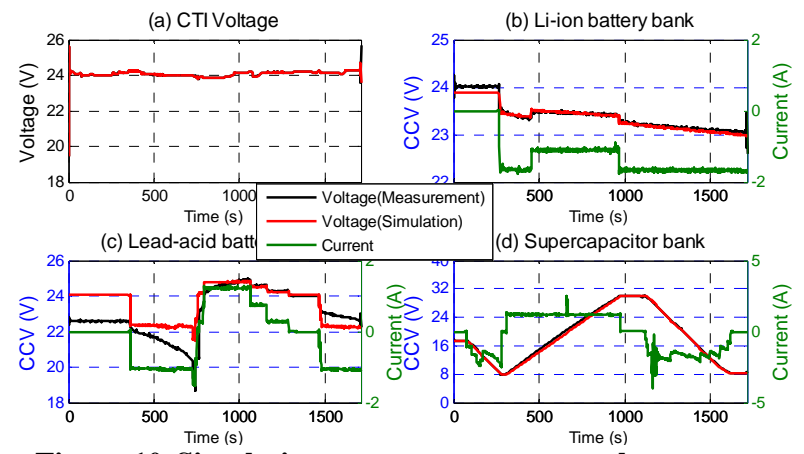

Figure 10. Simulation vs. measurement results.

\subsection{Household Application}

In this section we use SIMES to simulate the HEES system in a household application.

\subsubsection{Problem Background}

A major challenge in the electrical grid is the high peak consumption. On the generation side, utility companies can increase peak generation capacity, which requires a huge investment. However, the peak capacity is not fully utilized for most of the time [21] On the consumption side, users can perform demand side management, i.e., shift their demand from peak hours to off-peak hours, only if they're granted a certain profit [22]. Previous research work has shown that home users equipped with an energy storage system can achieve profit under certain pricing policies [23]. Here we consider a similar problem of daily household peak-shaving with a HEES system, which consists of a Li-ion battery bank and leadacid battery bank. We evaluate with the help of SIMES the total profit to the homeowner of installing such a system.

\subsubsection{Problem Formulation}

For simplicity, we assume there are only one peak period and one off-peak period, each with different unit price for the electrical energy. The simplest strategy to operate an energy storage system is to charge it during off-peak hours and discharge it during peak hours. Let $p_{h}, p_{l}$ denote the unit price for peak period and off-peak period, $E_{h}, E_{l}$ denote the energy supply to household electrical appliances during peak hours and the energy drawn from the grid during off-peak hours, respectively. The profit made by the HEES system is thusly the reduction on electric bills as:

$$
p_{h} E_{h}-p_{l} E_{l}
$$

We use SIMES to determine $E_{h}$ and $E_{l}$ for a given HEES system.

\subsubsection{Simulator Setup}

The SIMES setup is similar as in Section 5.1. A new main controller class is implemented to control the charging and discharging of two battery banks. The charging or discharging current is determined on the fly based on the SoC of each bank and the remaining time of the present period ( $t_{h}$ for peak and $t_{l}$ for off-peak):

$$
\begin{gathered}
I_{c}=Q(1-S o C) / t_{l} \\
I_{d}=Q \cdot S o C / t_{h}
\end{gathered}
$$

\subsubsection{Simulation Results}
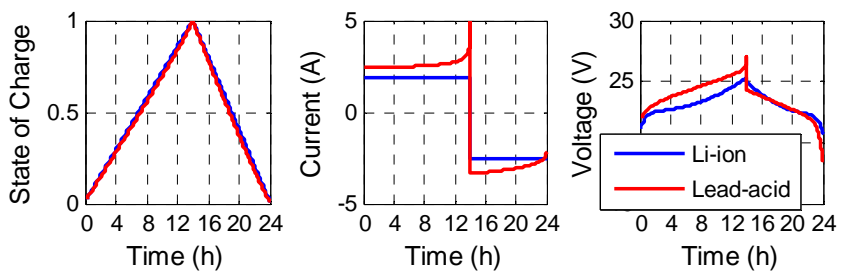

Figure 11. SoC, current and voltage of both banks.

The capacity of the Li-ion battery and lead-acid battery bank used in the simulation is 26Ah and 34Ah, respectively, with $24 \mathrm{~V}$ rated terminal voltage. SIMES simulates its operation for one day, which consists of a 14-hour off-peak period and a 10-hour peak period. Simulation results are reported in Figure 11. The runtime of the simulation is $0.07 \mathrm{~s}$. The total energy drawn from the grid is $1575 \mathrm{Wh}$ and the total energy supplied to the grid is 1266Wh. Therefore, for the above HEES system to make profit, the electricity price must satisfy:

$$
\frac{p_{h}}{p_{l}} \geq \frac{1575}{1266} \approx 1.24
$$

\subsection{Mobile Device Application}

We show that an online expert-based power management policy can be implemented and simulated using SIMES.

\subsubsection{Problem Background}

Power consumption is a primary concern in battery-powered devices. Their energy efficiency can be improved by reducing power loss in the power supply. A significant portion of batteries' power loss is due to the rate capacity effect, which can be greatly lowered by introducing supercapacitors as energy buffers [12].

We consider a mobile device powered by a HEES system which consists of a Li-ion battery bank and a supercapacitor bank. The mobile device frequently transitions between different power states. We focus on the management policy of the HEES system whose goal is to minimize the energy loss in the HEES system.

\subsubsection{Problem Formulation and Solution}

We formulate the problem of power management in the embedded HEES system as follows:

Given: At each time step $t_{k}$

1) Li-ion battery and supercapacitor bank's SoCs: $S o C_{b, k}$, $\mathrm{SoC}_{s, k}$;

2) Power demand of the mobile device: $P_{\text {load }, k}$;

3) The nominal input voltage of the mobile device: $V_{n}$

Find: Discharging current of both banks: $I_{b, k}, I_{s, k}$;

Minimize: Energy loss in the HEES system, defined as the difference between the total energy drawn from the HEES system and the actual energy delivered to the load device:

$$
E_{\text {loss }}=E_{\text {drawn }}-E_{\text {load }}
$$

Note that the voltage level of CTI is regulated to maintain the same as the nominal input voltage $V_{n}$ of the mobile device.

To solve the above problem, we first propose a heuristic management policy. The pseudo code of this policy is:

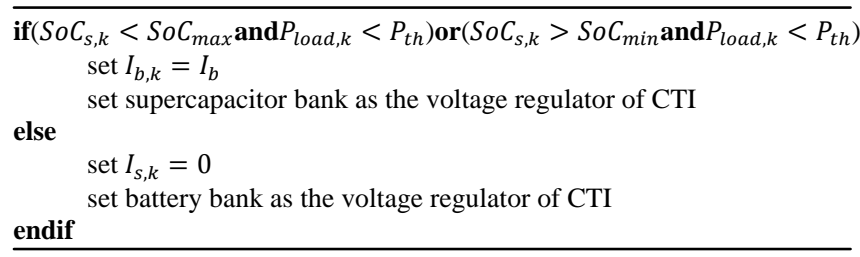


The above routine is implemented in an event-driven manner, i.e., it is called whenever there is a notable change on $P_{\text {load }}$ or $S o C_{S}$. The intuition behind this heuristic is: First, the battery's output current should be kept stable to minimize the energy loss due to rate capacity effect. Second, whenever $S o C_{s}$ and $P_{\text {load }}$ are both low, the battery is used to charge the supercapacitor. Third, whenever $S o C_{s}$ and $P_{\text {load }}$ are both high, the supercapacitor is used to provide power to the load together with the battery.

The performance of the above heuristic is based on the parameters (i.e., $S o C_{\min }, S o C_{\max }, P_{t h}, I_{b}$ ). Instead of determining the optimal parameters beforehand, we adopt an online expert-based approach: A set of experts are generated first, each implementing the same aforesaid heuristic management policy, but with different parameters. At each decision epoch, we (i) select the expert with highest rating $r_{i}$ to activate until the next decision epoch and (ii) update the rating $r_{i}$ of the active expert $i$ based on the energy efficiency of the HEES system during the time when expert $i$ is active:

where $\alpha$ is the learning rate.

$$
r_{i}=(1-\alpha) r_{i}+\alpha \frac{E_{\text {load }}}{E_{\text {drawn }}}
$$

\subsubsection{Simulator Setup}

A different DC-DC converter model is used, and a new main controller class is added to implement the above expert-based approach. All other models are the same as in Section 5.1 but with different parameters such as storage banks' capacity.

\subsubsection{Simulation Results}

To generate the load demand profile, a synthesized continuoustime Markov model is used. The states of this Markov process corresponds with the mobile device's power states. The power consumption of each power state is shown in Table 1.

Table 1. Power Consumption of Each State.

\begin{tabular}{|c|c|c|c|c|c|}
\hline State \# & 1 & 2 & 3 & 4 & 5 \\
\hline Power & $3.0 \mathrm{~W}$ & $2.4 \mathrm{~W}$ & $1.5 \mathrm{~W}$ & $0.3 \mathrm{~W}$ & $0 \mathrm{~W}$ \\
\hline
\end{tabular}

(a) Overall Power Consumption

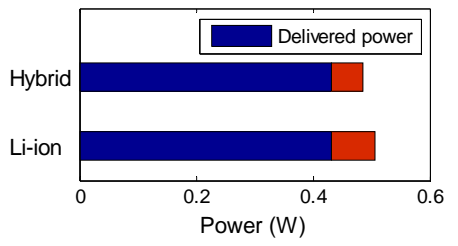

(b) Power Loss

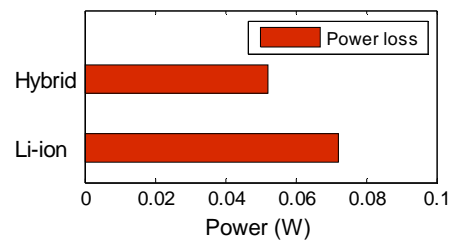

Figure 12. Power consumption of the mobile device.

To evaluate the merit of the HEES system, we compare its performance with a baseline system which is a Li-ion battery-only supply. We simulate both systems in SIMES. The runtimes of both simulations are 0.56s and 0.29s, respectively. As shown in Figure 12 , hybrid battery/supercapacitor system reduces overall power consumption by $4 \%$ and power loss by $28 \%$.

\section{Conclusion}

This paper presents SIMES, a simulator for HEES systems. SIMES performs fast and accurate simulation for HEES systems ranging from large-scale household applications to small-scale mobile device applications. It achieves less than 3\% error with a runtime of 1.1s compared to a half-hour prototype measurement result. We then apply SIMES to two applications: In the household application, SIMES is used to determine the efficiency of a gridconnected HEES system in order to study its profitability. In the mobile device application, SIMES is used to test the performance of an online HEES system management policy.

\section{Acknowledgement}

This work is supported in part by the Software and Hardware Foundations program of the NSF's Directorate for Computer \& Information Science \& Engineering, the Center for Integrated Smart Sensors funded by the Ministry of Science, ICT \& Future Planning as Global Frontier Project (CISS2012054193). The SPORT lab at USC and ICT at SNU provides research facilities for this study.

\section{References}

[1] H. T. Odum, "Energy quality and carrying capacity of the earth," Tropical Ecology, 1976.

[2] M. Pedram, N. Chang, Y. Kim, and Y. Wang, "Hybrid electrical energy storage systems," Proc. of ISLPED, Aug. 2010.

[3] F. Koushanfar, "Hierarchical hybrid power supply networks," Proc. of DAC, Jun. 2010.

[4] A. Mirhoseini, and F. Koushanfar. "HypoEnergy: Hybrid supercapacitor-battery power-supply optimization for Energy efficiency,” Proc. of DATE, Mar. 2011.

[5] A. Khaligh, and Z. Li, "Battery, ultracapacitor, fuel cell, and hybrid energy storage systems for electric, hybrid electric, fuel cell, and plugin hybrid electric vehicles: State of the art," IEEE TVT, Vol. 59, No. 6, 2010.

[6] A. Khaligh, et al., "Digital control of an isolated active hybrid fuel cell/Li-ion battery power supply,” IEEE TVT, Vol. 56, No. 6, 2007.

[7] Q. Xie, et al, "Charge allocation for hybrid electrical energy storage systems," Proc. of CODES, Oct. 2011.

[8] Y. Wang, Y. Kim, Q. Xie, N. Chang, and M. Pedram, "Charge migration efficiency optimization in hybrid electrical energy storage (HEES) systems," Proc. of ISLPED, 2011.

[9] Y. Wang, et al, "Multiple-source and multiple-destination charge migration in hybrid electrical energy storage systems," Proc. of DATE, Mar. 2012.

[10] Q. Xie, et al, "Charge replacement in hybrid electrical energy storage systems," Proc. of ASP-DAC, Jan. 2012.

[11] D. Zhu, et al, "Maximizing Return on Investment of a Grid-Connected Hybrid Electrical Energy Storage System,” Proc. of ASP-DAC, Jan. 2013.

[12] D. Shin, Y. Wang, N. Chang, and M. Pedram, "Battery-supercapacitor hybrid system for high-rate pulsed load applications," Proc. of DATE, Mar. 2011.

[13] F. Koushanfar, and A. Mirhoseini. "Hybrid heterogeneous energy supply networks,” Proc. of ISCAS, May. 2011.

[14] Q. Xie, et al, "State of health aware charge management in hybrid electrical energy storage systems," Proc. of DATE, Mar. 2012.

[15] LTC3789 Datasheet: http://www.linear.com/product/LTC3789.

[16] A. Mirhoseini, and F. Koushanfar "Learning to manage combined energy supply systems,” Proc. of ISLPED, Aug. 2011

[17] R. K. Sharma, and K. Kudo "Integrated management of energy storage for sustainable operation of energy microgrids". Proc. of the ASME 2011 IMECE, Nov. 2011

[18] J. Cao, and A. Emadi, "A new battery/ultra-capacitor hybrid energy storage system for electric, hybrid and plug-in hybrid electric vehicles," Proc. of VPPC, Sep. 2009.

[19] Y. Kim, et al, "Networked architecture for hybrid electrical energy storage systems," Proc. of DAC, Jun. 2012.

[20] D. Linden and T. B. Reddy, Handbook of Batteries. McGrew-Hill Professional, 2001.

[21] E. M. Lightner, and S. E. Widergren, "An orderly transition to a transformed electricity system,” IEEE TSG, Vol. 1, No. 1, 2010.

[22] S. Yue, J. Chen, Y. Gu, C. Wu, and Y. Shi, "Dual-pricing policy for controller-side strategies in demand side management," Proc. of SmartGridComm, Dec. 2011.

[23] N. S. Wade, P. C. Taylor, P. D. Lang, and P. R. Jones, "Evaluating the benefits of an electrical energy storage system in a future smart grid," Energy policy, Vol. 38, No. 11, 2010.

[24] http://www.panasonic.com/industrial/includes/pdf/Panasonic_VRLA LC-R123R4P.pdf

[25] http://www.grinninglizard.com/tinyxml/

[26] http://qt-project.org/

[27] http://www.workslikeclockwork.com/index.php/components/qtplotting-widget/ 
